\title{
Quantum effects of hydrogen nuclei on a structure and a dynamical rearrangement of hydrogen-bond networks
}

\author{
Kim Hyeon-Deuk ${ }^{1,2}$ \\ 1. Department of Chemistry, Kyoto University, Kyoto, JAPAN, 606-8502 \\ 2. Japan Science and Technology Agency, PRESTO, 4-1-8 Honcho, Kawaguchi, \\ Saitama, JAPAN, 332-0012
}

\begin{abstract}
To understand anomalous energetics and dynamics in water, nuclear quantum effects such as zero-point energy and delocalization of wave packets(WPs) representing water hydrogen atoms should be essential. However, since quantum calculations of many-body dynamics are highly complicated in general, none has yet directly viewed the quantum WP dynamics of hydrogen atoms in liquid water. Our semiquantum molecular dynamics simulation for the first time made it possible to observe the hydrogen WP dynamics in liquid water. Here, we demonstrate that the microscopic WP dynamics are closely correlated to and play key roles in the dynamical rearrangements in the hydrogen-bond network(HBN) of liquid water. Especially, the memory loss of the dipolar angle formed by two waters is accelerated with the nuclear quantum effect introduced by the nuclear WP, rationalizing the faster memory decay of the HBN in the quantum liquid water. We found the quantum effects of hydrogen atoms on mesoscopic liquid water behaviors such as intermittent collective motions associated with the rearrangement of HBN and the concomitant fluctuation and relaxation. Our results will provide new physical insights on HBN dynamics in water whose significance is not limited to the pure liquid dynamics but also for understanding chemical and biological reactions in liquid water.
\end{abstract}

Keywords: Semiquantum Molecular Dynamics Simulation, Nuclear Quantum Effects, Liquid Water, Condensed Phase Quantum Dynamics 


\section{Introduction}

Chemical reactions taking place in water are essential for many important processes in electrochemistry, environmental, pharmaceutical, and biological sciences. It is recognized that the unique and anomalous properties of water stem from its dynamics of hydrogen-bond network(HBN). Many attempts have been conducted to clarify how liquid water is dynamically organized via the HBN. Computer simulation has been a strategy of particular importance in this field. A number of empirical potentials have been developed toward better agreement with experiments, with the parameters calibrated for use in full classical water(FCW) molecular dynamics(MD) and Monte Carlo simulations. $(1 ; 2 ; 3 ; 4)$ The classical MD is nowadays very straightforward, and is routinely used to study complex chemical problems involving many thousands of particles.(3) In contrast, quantum mechanical simulations are yet unestablished for many-body dynamics in condensed phases, despite that the nuclear quantum effects such as zero-point energy(ZPE), delocalization of WPs describing water hydrogen atoms, etc. are envisaged to be essential in the HBN dynamics. Although considerable progress has been made in the development of exact quantum-mechanical methods, they are yet applicable to relatively small systems and short times, and only the static spacial and momentum distributions were obtained. In order to capture such quantum effects in many degrees of freedom, a number of approaches exploiting semiquantum and mixed quantum-classical simulations have been proposed and applied in recent years. The path-integral Monte Carlo(PIMC) method was used to assess the effect of quantum-mechanical fluctuations on a liquid structure $(5 ; 6)$, and the centroid molecular dynamics(CMD) method was proposed to study water dynamics through the equilibrium correlation functions and mean square displacement.(7) However, none has yet directly viewed quantum dynamics of water hydrogen atoms in condensed bulk water and their effects on mesoscopic behaviors such as intermittent collective motions associated with a rearrangement of HBNs and the concomitant fluctuation and relaxation. $(1 ; 8)$ We for the first time report a direct simulation study of hydrogen WP dynamics in liquid water, and suggest its key roles in a mesoscopic dynamical rearrangement of HBNs.

\section{Simulation Method}

In order to take account of the nuclear quantum aspects such as the WP delocalizing and the ZPE effects in the liquid water dynamics, the hydrogen 
atoms are described by $N$-independent symmetric $3 \mathrm{D}$ squeezed coherent state Gaussian WP basis functions, $\Psi\left(\left\{x_{i}, y_{i}, z_{i}\right\}, t\right)=\prod_{i=1}^{N} \Psi_{i}\left(x_{i}, y_{i}, z_{i}, t\right)$, where

$$
\begin{aligned}
\Psi_{i}\left(x_{i}, y_{i}, z_{i}, t\right) & =N_{i} \exp \left[A_{i}(t)\left\{\left(x_{i}-q_{x_{i}}(t)\right)^{2}+\left(y_{i}-q_{y_{i}}(t)\right)^{2}+\left(z_{i}-q_{z_{i}}(t)\right)^{2}\right\}\right. \\
& \left.+i p_{x_{i}}(t)\left\{x_{i}-q_{x_{i}}(t)\right\}+i p_{y_{i}}(t)\left\{y_{i}-q_{y_{i}}(t)\right\}+i p_{z_{i}}(t)\left\{z_{i}-q_{z_{i}}(t)\right\}\right] .
\end{aligned}
$$

$q_{\alpha_{i}}(t)$ and $p_{\alpha_{i}}(t)$ with $\alpha_{i}=x_{i}, y_{i}$ or $z_{i}$ are the 3D Cartesian coordinates of the WP center and their momenta, respectively. (For simplicity, $\hbar=1$ and all the coordinates are mass-scaled.) $A_{i}$ is defined by $A_{i}(t)=(-1+$ $\left.2 i \rho_{i}(t) \Lambda_{i}(t)\right) / 4 \rho_{i}^{2}(t)$, where $\rho_{i}(t)$ and $\Lambda_{i}(t)$ correspond to the WP width and its momentum, respectively. $\quad N_{i}=\left(2 \pi \rho_{i}^{2}(t)\right)^{-3 / 4}$ is the normalization factor. The WP function (1) is specified by the time-dependent parameters $\left\{q_{\alpha_{i}}(t), p_{\alpha_{i}}(t), \rho_{i}(t), \Lambda_{i}(t)\right\}$ to be determined through the time-dependent variational principle of the action integral. $(9 ; 10 ; 11 ; 12 ; 13 ; 14)$ The resulting equations of motions(EOM) which we have to solve finally become

$$
\begin{array}{ll}
\dot{q}_{\alpha_{i}}=\frac{p_{\alpha_{i}}}{m_{i}}, & \dot{p}_{\alpha_{i}}=-\frac{\partial \sum_{i<j}^{N}\left\langle V_{i j}\left(x_{i j}, y_{i j}, z_{i j}\right)\right\rangle}{\partial q_{\alpha_{i}}} \\
\dot{\rho}_{i}=\frac{\Lambda_{i}}{m_{i}}, & \dot{\Lambda}_{i}=-\frac{\partial \sum_{i<j}^{N}\left\langle V_{i j}\left(x_{i j}, y_{i j}, z_{i j}\right)\right\rangle}{3 \partial \rho_{i}}+\frac{\hbar^{2}}{4 m_{i} \rho_{i}^{3}},
\end{array}
$$

for $i=1,2, \cdots, N$. Here, the intra- and intermolecular potential functions $V_{i j}\left(x_{i j}, y_{i j}, z_{i j}\right)$ are averaged as $\langle X\rangle \equiv\langle\Psi, t|X| \Psi, t\rangle$ (and the mass $m_{i}$ and $\hbar$ have been retrieved). Our semiquantum water(SQW) program requires only $2 N$ additional EOM for the auxiliary coordinates and momenta representing the WP widths of hydrogen atoms. The 3D SQW MD simulation was developed by expanding the potentials $V_{i j}\left(x_{i j}, y_{i j}, z_{i j}\right)$ around $\left\langle x_{i}\right\rangle,\left\langle y_{i}\right\rangle$ and $\left\langle z_{i}\right\rangle$ for hydrogen atoms. $(9 ; 10)$ The dynamics of oxygen atoms are also influenced by the additional non-classical forces from the semiquantum hydrogen atoms. With the explicit extended potential functions, we can perform the SQW dynamics simulations of 1024 water molecules solving the EOM (2) and (3). The extra computational costs for SQW over full classical water(FCW) are only marginal, about 20\%. Nevertheless, our SQW simulation reproduced the major properties of the PIMC and CMD methods as was reported in our previous paper.(9) Our SQW method could be especially effective in biosimulations where a large number of solvating water molecules are often needed to realize stable simulations. 


\section{Results and Discussions}

Figure 1 shows power spectra of kinetic and potential energies of WPs describing the semiquantum hydrogen atoms (the left two panels). The power spectrum is defined as the absolute square of Fourier transformed energy dynamics, $\left|\int E(t) \exp (i \omega t) d t\right|^{2}$. The power spectra of the low frequency region are both well-fitted by $1 / f^{2}$, while the higher frequency spectra exhibit the complicated behavior. This is quite different from the classical parts of kinetic, potential and intramolecular energies in the same semiquantum water(SQW) simulation, where the entire spectra can be fitted by single functions $1 / f^{x}$ with the exponents $0.5<x<1.5$ (the right three panels). The power spectra demonstrate that, although the classical parts exhibit multitimescale dynamics as was reported by previous works(8), the low frequency energy dynamics of WPs are rather of single-timescale and not strongly influenced by the classical energy fluctuations.

To study dynamics of the structure, we define the time-dependent radial distribution functions(RDFs) as

$$
g(r, t)=\frac{n(r, t)}{4 \pi r^{2} d r n_{0}},
$$

where $n(r, t)$ represents the number of oxygen atoms between shells of radii $r$ and $r+d r$ from a representative oxygen atom (to be called $i$ th oxygen atom), and $n_{0}$ is the oxygen number density of the whole system. The RDF dynamics directly reflects the liquid structure rearrangement around the oxygen atom. Figure 2 shows the time-dependent maximum peak positions of the RDFs for pairs of the $i$ th oxygen atom and the surrounding other 1023 oxygen atoms. The panels are different in the time-resolution for averaging as indicated beside each panel. With the finer time-resolution, the maximum peak positions for SQW and FCW both fluctuate randomly reflecting the short-time thermal modulations. When the time-resolution is coarse-grained, the random thermal fluctuations are smeared out and the time-dependent maximum peak position exhibits a behavior of transitions between well-structured and less-structured states. This implies that the water structure in the meso-timescale have two typical configurations at fully coordinated and distorted local HBNs. It supports the mixture model recently reported by X-ray experiments. $(15 ; 16 ; 17)$ We also mention that the distorted structure for SQW is less structured than that for FCW, reflecting the smoothed free energy landscape due to the ZPE of the semiquantum hydrogen atoms. $(9 ; 10 ; 11 ; 12)$ 
Figure 3 demonstrates that the hydrogen quantum effects influence the hydrogen bond dynamics. We calculated the number of water molecules hydrogen-bonded to the $i$ th water molecule as a function of time, $N_{\mathrm{HB}}(t)$. The common geometrical criterion for hydrogen bonds was used(3), i.e., if the distance between two oxygen atoms, $r_{\mathrm{OO}}(t)$, is less than $3.5 \AA$ and the $\mathrm{OH} \cdots \mathrm{O}$ angle is greater than $150 \mathrm{deg}$, the water pair is considered as hydrogen bonded. The evolution of $N_{\mathrm{HB}}(t)$ directly reflects the HBN dynamics.(1) The upper panel of Fig.3 shows the normalized time correlation functions(TCFs) $\left\langle N_{\mathrm{HB}}(t) N_{\mathrm{HB}}(0)\right\rangle /\left\langle N_{\mathrm{HB}}^{2}(0)\right\rangle$. The TCF for SQW decreases faster than the TCF for FCW, indicating that the quantum effect of hydrogen atoms accelerates the memory decay of the HBN. We further show the TCF of the angular coordinate $\phi(t)$ which is defined as an angle formed by dipole vectors of two water molecules (dipolar angle). The angular TCF exhibits the similar tendency: The memory of the dipolar angle decays faster with the nuclear quantum effects than without the nuclaer quantization, rationalizing the faster memory decay of the HBN in the quantum liquid water. These accelerated memory decays are in accord with the experimentally observed ultrafast memory loss in liquid water.(18) Figure 3 also shows that the kink-like ultrafast ( $2 \mathrm{fs}$ ) dynamics appears both in the HBN and dipolar angle dynamics; the disappearance of the HBN kink in the SQW case partially depends on the definition of a hydrogen bond since the current kink dynamics is ultrafast. Because the TCFs of the dipolar angle exhibit the kink, the current water dynamics clearly involves a kink-like motion related to a molecular rotation.

To further demonstrate the significance of the nuclear quantum dynamics, we show in Fig.4 the time evolutions of the WP width of the two hydrogen atoms $\rho_{i, \mathrm{H}_{1}}(t)+\rho_{i, \mathrm{H}_{2}}(t)$ of the $i$ th water molecule, the average $\mathrm{OH}$ bond length $\left(r_{i, \mathrm{OH}_{1}}(t)+r_{i, \mathrm{OH}_{2}}(t)\right) / 2$ of the $i$ th water molecule, and the number of hydrogen bonds $N_{\mathrm{HB}}(t)$ to the $i$ th water molecule. All the data were time averaged over every $1.25 \mathrm{ps}$ to view the meso-timescale dynamics. As indicated by the circles, notable correlations among these three variables are observed. When the WP width increases, both the $\mathrm{OH}$ bond length and the hydrogen bond number decrease. Although these correlations are not complete, reflecting complicated fluctuations with coupling to other variables, they are seen not only in the shown timescale in Fig.4 but also throughout our SQW MD simulations. The significant correlations are observed particularly in the characteristic regions where the dynamics turn over, as marked by the circles. As the WP width grows, the $\mathrm{OH}$ bond length becomes small 
due to the excluded volume effect and repulsive force induced by the WP semiquantum hydrogen atoms. The decreasing in the $\mathrm{OH}$ bond length leads to less structured liquid, and results in weakening the HBN. In other words, the quantum WP delocalizations distort the hydrogen-bond structure and induce higher mobility of waters to move out of the HBN like a cage breakout. We should note that, although the quantitative average number of hydgogen bonds is still controversial $(15 ; 16 ; 17)$, the final conclusion does not affect our qualitative picture.

Figure 5 displays the WP dynamics (left column) and the corresponding mean-square displacements (right column) of the $i$ th oxygen atom, $\mid \mathbf{r}_{i}(t)-$ $\left.\mathbf{r}_{i}(0)\right|^{2}$, where $\mathbf{r}_{i}(t)$ denotes the position vector of the $i$ th oxygen atom at time $t$. The arrows in the right column mark the significant changes in the mean-square displacement which are considered to represent the mesoscale motion of the $i$ th molecule such as cage breakout. These large displacements are always accompanied by the WP broadening, as the arrows in the left column indicate. This correlation is observed for both the increase and decrease of the $\mathbf{r}_{i}(t)$ displacements. The WP broadening weakens the hydrogen bond around the oxygen atom, and thus induces the collective changes of the configuration. This provides a physically intuitive picture for the increased diffusion constant and the reduced structure reported in the semiquantum water simulations. $(5 ; 6 ; 7 ; 9)$

In summary, we for the first time directly viewed the quantum dynamics of hydrogen atoms in bulk water. We found the significant correlations between the hydrogen WP dynamics and the HBN dynamics, and demonstrated their actual influences on the collective HBN rearrangements. The faster memory decay of the HBN with the nuclear quantum effect was explained by the accelerated ultrafast memory loss of the dipolar angle between waters. The results will provide new physical insights on the HBN dynamics in water which are important not only for describing the pure liquid water dynamics but also for understanding chemical reactions and biomolecule dynamics in water.

\section{Acknowledgments}

KHD acknowledge the financial supports from JST (PRESTO), and Grantin-Aids for Scientific Research from Japan Society for the Promotion of Science (KAKENHI), Grant No. 24750016. 


\section{References}

[1] D. Laage, J. T. Hynes, Science 311 (2006) 832-835.

[2] L. X. Dang, B. M. Pettitt, Journal of Physical Chemistry 91 (1987) 3349-3354.

[3] Y. Wu, H. L. Tepper, G. A. Voth, Journal of Chemical Physics 124 (2006) 024503.

[4] T. Yagasaki, S. Saito, Journal of Chemical Physics 128 (2008) 154521.

[5] R. A. Kuharski, P. J. Rossky, Journal of Chemical Physics 82 (1985) $5164-5177$.

[6] A. Wallqvist, B. J. Berne, Chemical Physics Letters 117 (1985) 214219.

[7] F. Paesani, W. Zhang, D. A. Case, T. E. Cheatham, G. A. Voth, Journal of Chemical Physics 125 (2006) 184507.

[8] M. Sasai, I. Ohmine, R. Ramaswamy, Journal of Chemical Physics 96 (1992) 3045-3053.

[9] K. Hyeon-Deuk, K. Ando, Journal of Chemical Physics 131 (2009) 064501.

[10] K. Hyeon-Deuk, K. Ando, Journal of Chemical Physics 132 (2010) 164507.

[11] J. Ono, K. Hyeon-Deuk, K. Ando, International Journal of Quantum Chemistry 113 (2013) 356-365.

[12] K. Hyeon-Deuk, K. Ando, Chemical Physics Letters 532 (2012) 124130.

[13] K. Ando, Journal of Chemical Physics 121 (2004) 7136-7143.

[14] K. Ando, Journal of Chemical Physics 125 (2006) 014104.

[15] T. Tokushima, Y. Harada, O. Takahashi, Y. Senba, H. Ohashi, L. G. M. Pettersson, A. Nilsson, S. Shin, Chemical Physics Letters 460 (2008) 387-400. 
[16] P. Wernet, D. Nordlund, U. Bergmann, M. Cavalleri, M. Odelius, H. Ogasawara, L. A. Naslund, T. K. Hirsch, L. Ojamae, P. Glatzel, L. G. M. Pettersson, A. Nilsson, Science 304 (2004) 995-999.

[17] J. D. Smith, C. D. Cappa, K. R. Wilson, B. M. Messer, R. C. Cohen, R. J. Saykally, Science 306 (2004) 851-853.

[18] M. L. Cowan, B. D. Bruner, N. Huse, J. R. Dwyer, B. Chugh, E. T. J. Nibbering, T. Elsaesser, R. J. D. Miller, Nature 434 (2005) 199-202. 


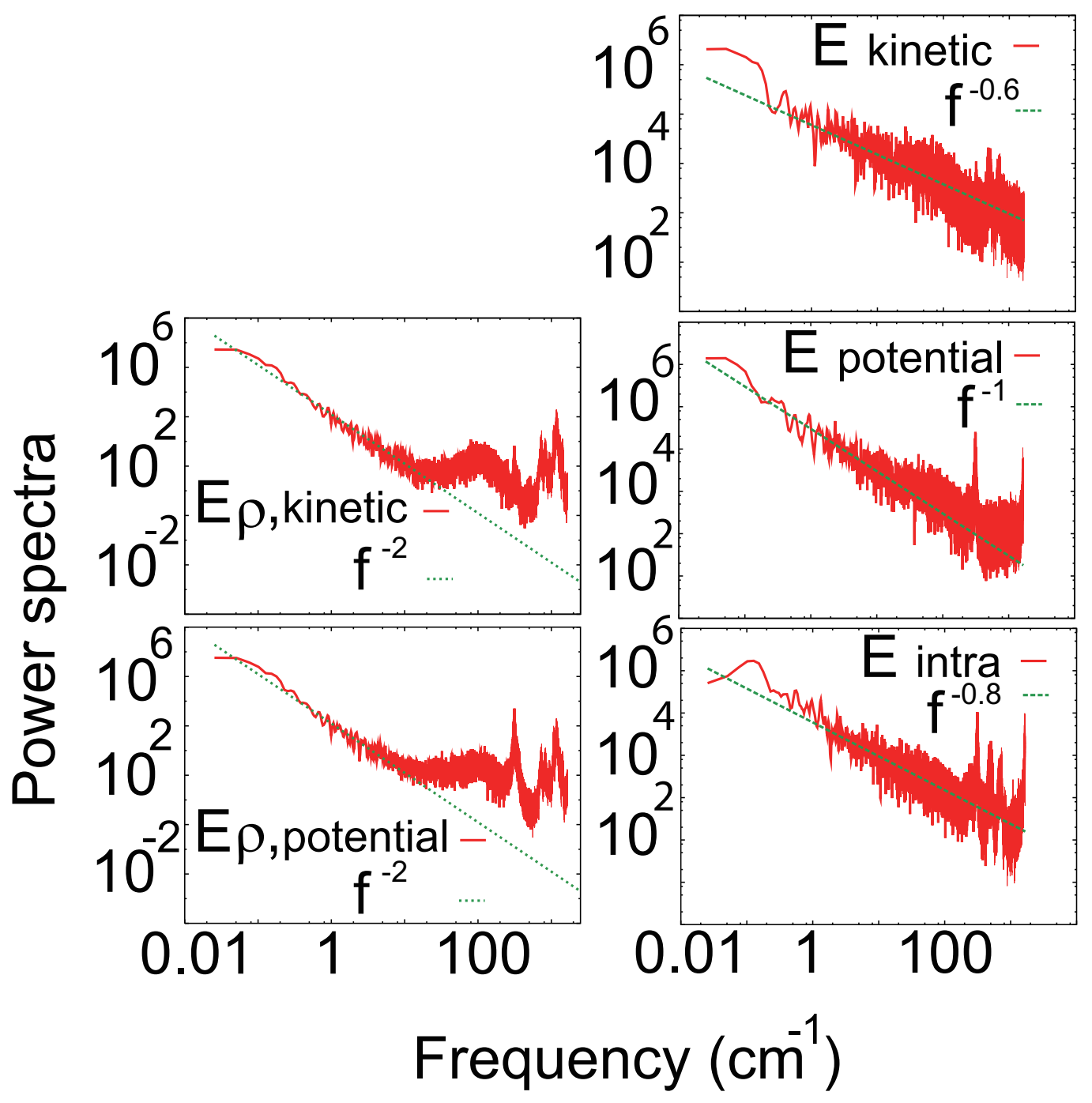

Figure 1: Power spectra of the kinetic and potential energies of WPs describing semiquantum hydrogen atoms, and the classical parts of kinetic, potential and intamolecular energies. The power spectra of the WP kinetic and potential energies show a simple decay $1 / f^{2}$ in the lower frequency region, whereas those of the classical energy parts are all reproduced by the functions $1 / f^{x}$ with the exponents $0.5<x<1.5$. Although the classical energy dynamics follow the multi-timescale dynamics, the WP energy dynamics are not strongly coupled to them and exhibit the single-timescale dynamics. 


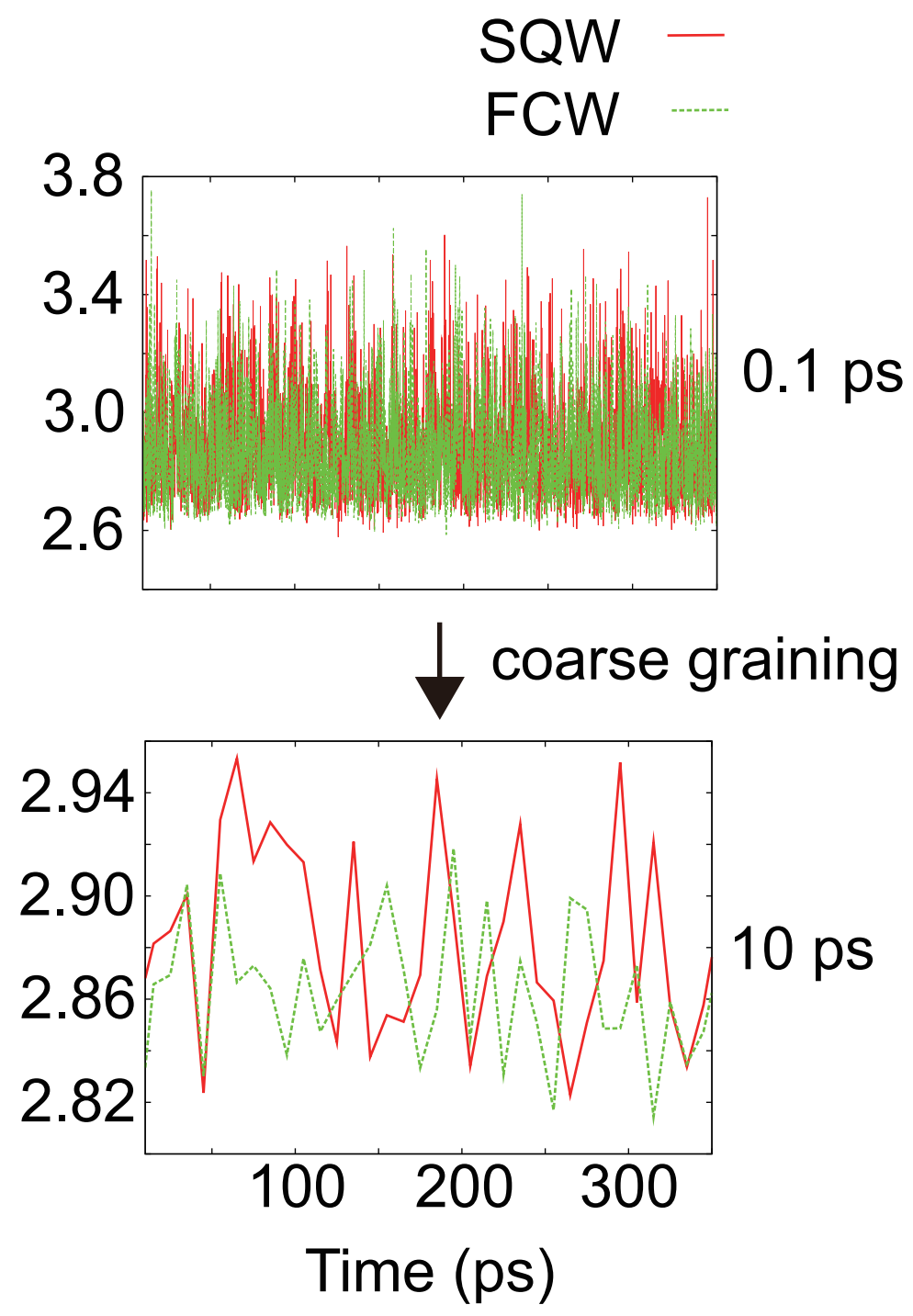

Figure 2: Averaged dynamics of the maximum peak position of the RDF around a representative oxygen atom. The time-resolutions for averaging are displayed beside each panel. The red and green lines express the semiquantum and classical results, respectively. With coarse-graining the time-resolution, the random thermal fluctuations disappear and the typical two-state configurations appear. The distorted structure for SQW is less structured than that for FCW. 

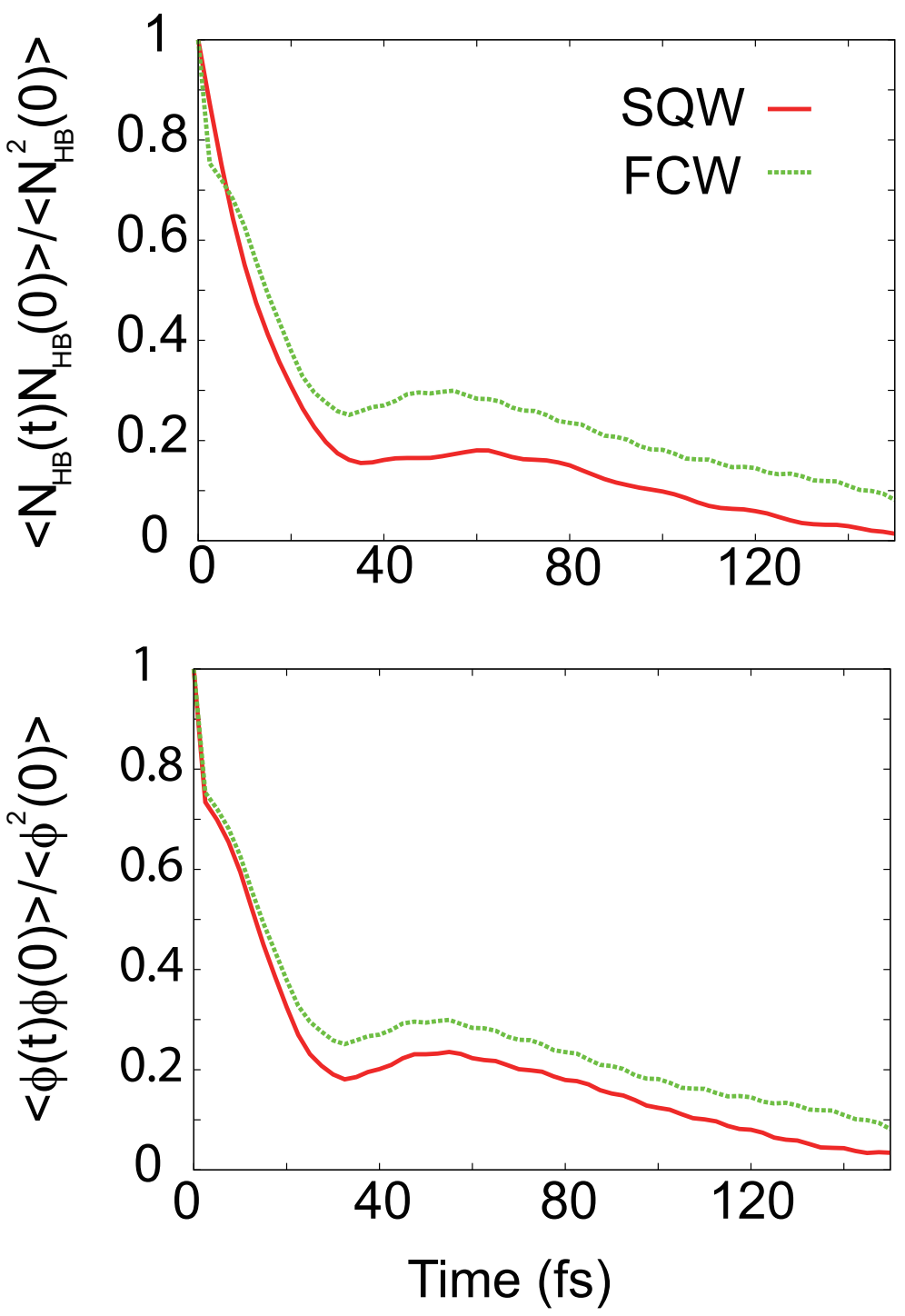

Figure 3: Dynamics of the number of hydrogen bonds $N_{\mathrm{HB}}(t)$ around a representative water molecule and the dipolar angle $\phi(t)$ between two water molecules. Each panel shows the normalized TCFs of $N_{\mathrm{HB}}(t)$ and $\phi(t)$ for SQW (red line) and FCW (green line). The faster decay in SQW indicates the accelerated memory loss due to the quantum hydrogen atoms. 


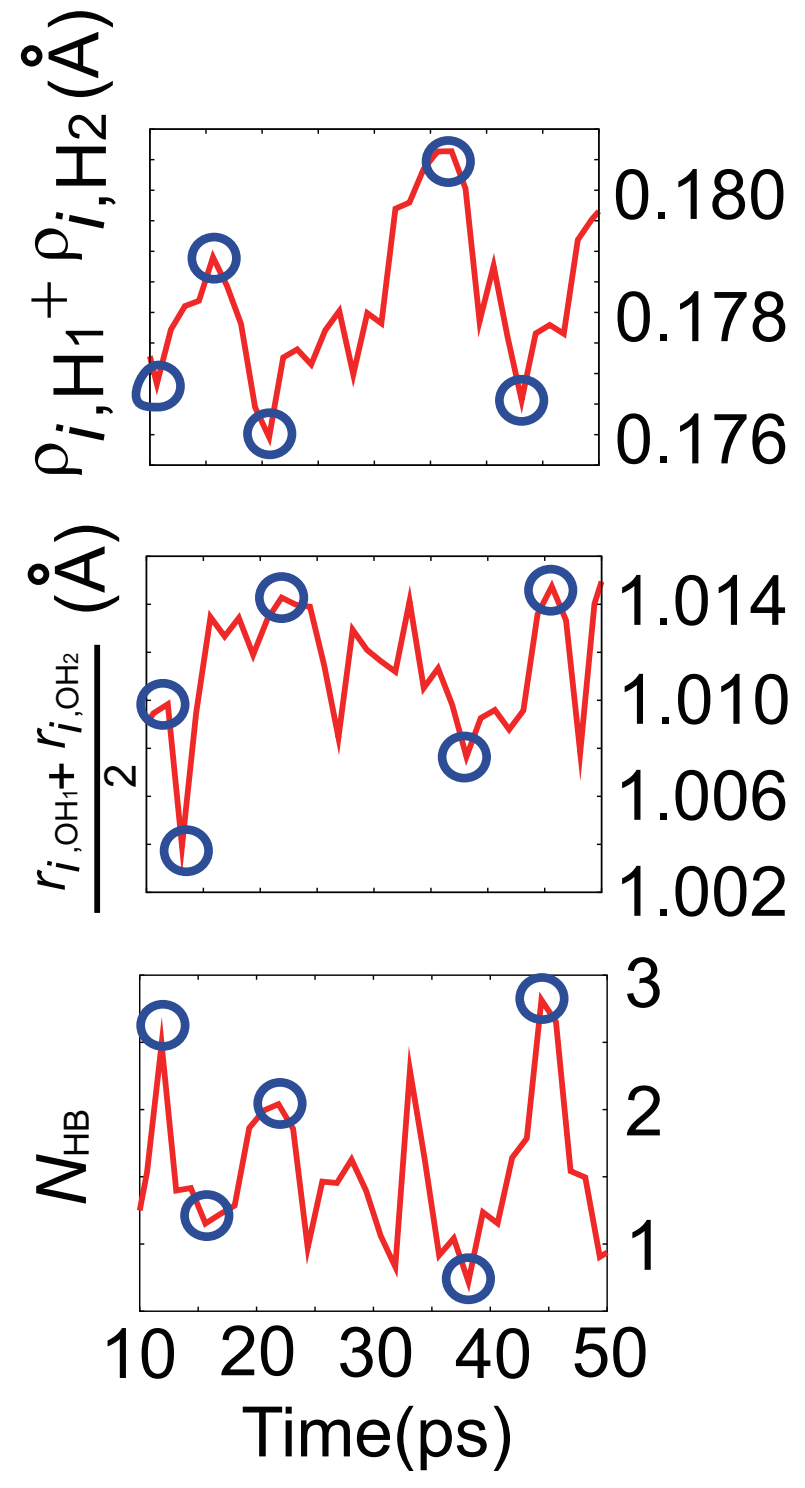

Figure 4: Dynamics of the WP width (upper), the average $\mathrm{OH}$ bond length (middle), and the number of hydrogen bonds (lower) for a representative water molecule. The circles mark notable correlations among these three variables in the characteristic regions where their dynamics turn over. 


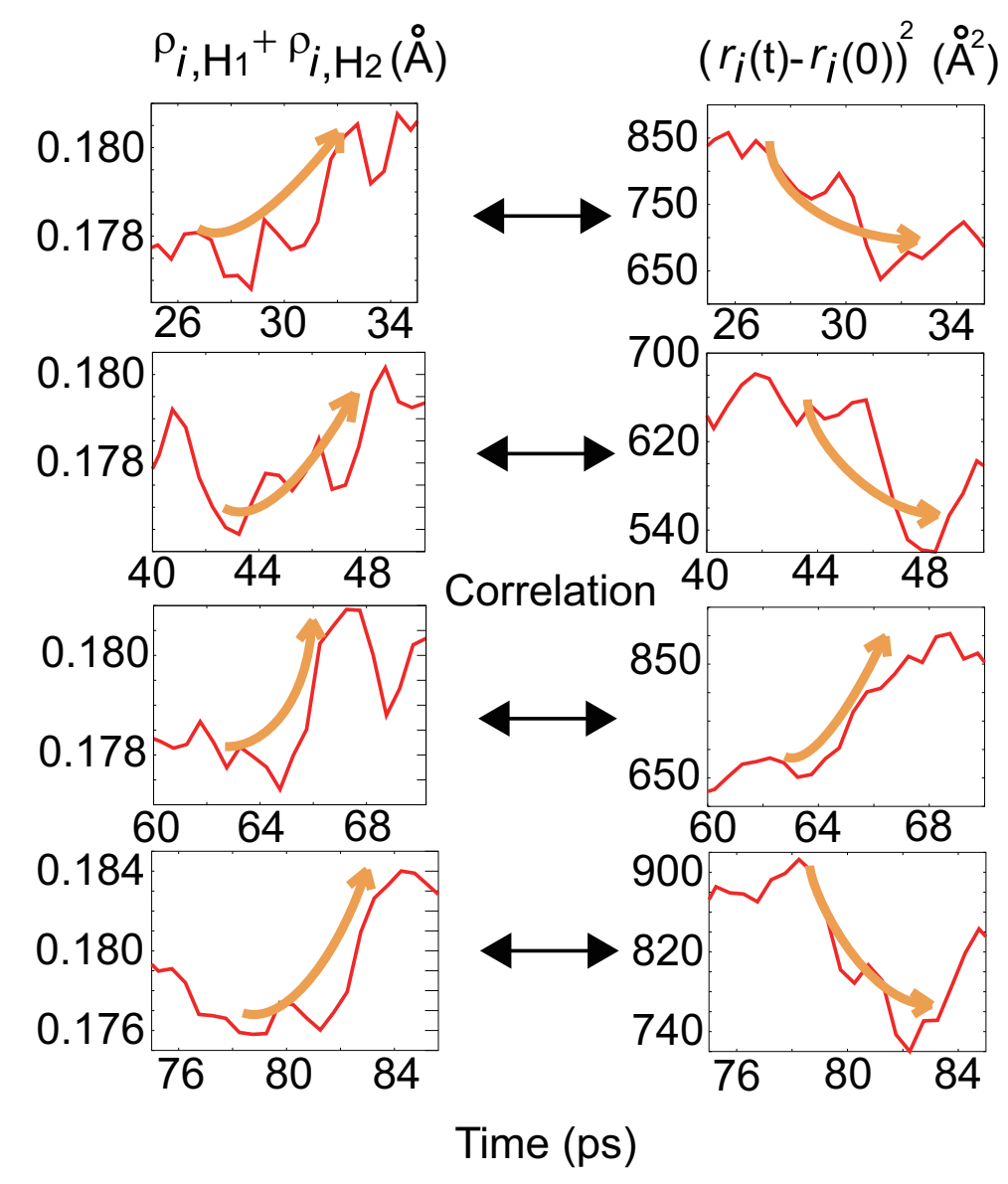

Figure 5: Dynamics of the hydrogen WP width(left column) and the mean-square displacement of the oxygen atom (right column) for a representative water molecule. The arrows indicate that the significant changes in the mean-square displacement are always accompanied by the WP broadening. 


\section{Quantization of Hydrogen Nucleus}

Classical Particle

Semiquantum Gaussian WP
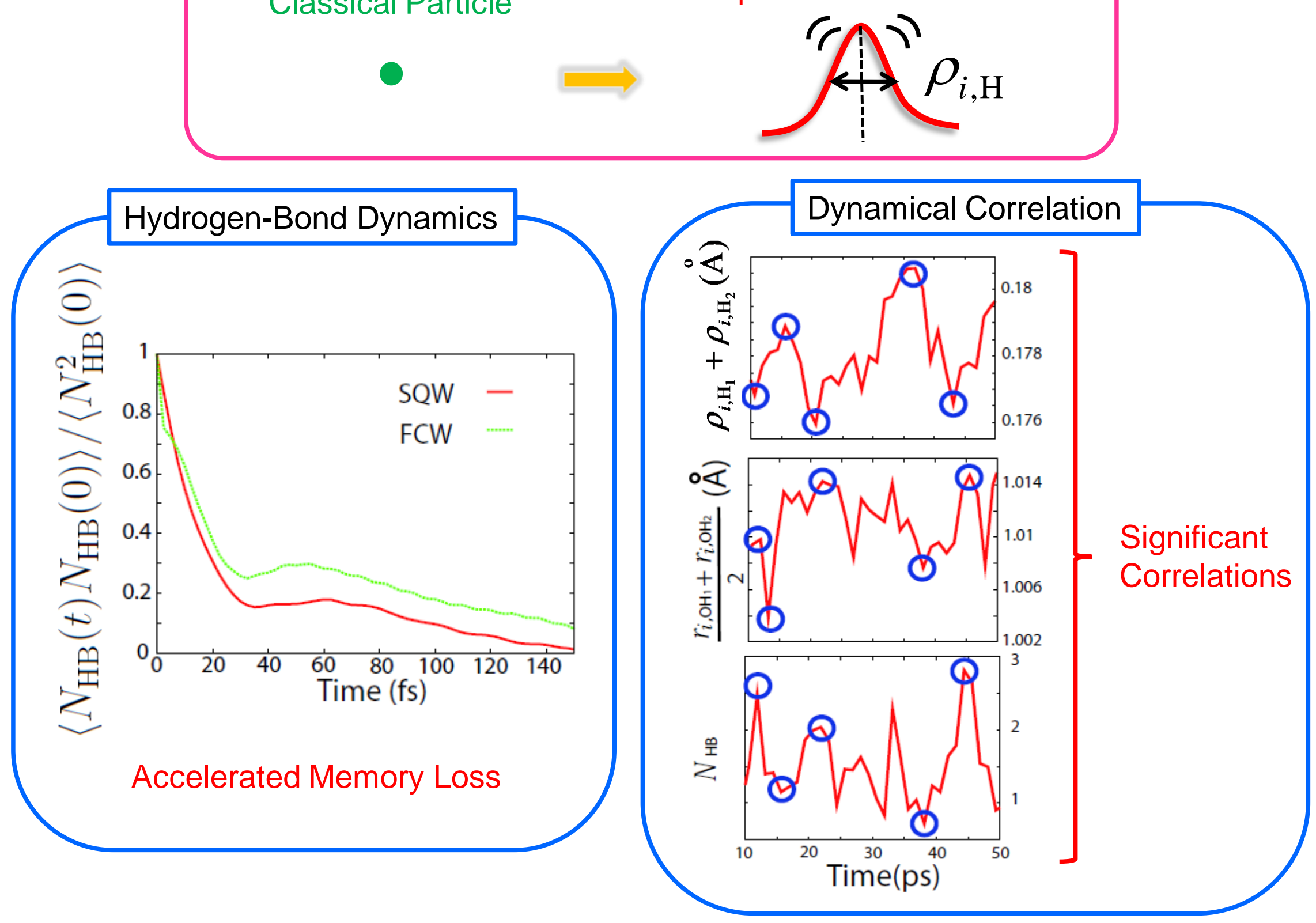